\title{
GUÍA PARA LA GENERACIÓN DE PLANES DE MANTENIMIENTO BASADOS EN ISO 9001: 2008 Y ANSI/ISA 88, 95. CASO DE ESTUDIO: PLANTA TRILLADORA DE CAFÉ PERGAMINO
}

\section{GUIDE FOR THE GENERATION OF MAINTENANCE PLANS BASED ON ISO 9001:2008 AND ANSI/ISA 88, 95. STUDY CASE: THRESHER PLANT OF PARCHMENT COFFE}

\author{
Ing. José Wilmer Majin Erazo*, Ing. Hernan Dario Gomez Chicue * \\ MSc. Juan Fernando Florez Marulanda* \\ *Institución 1, Facultad de Ingeniería Electrónica y Telecomunicación, Grupo de \\ Automática Industrial. \\ Calle 5 No 4-70, Popayán, Cauca, Colombia. \\ Teléfono y Fax, +57 (2) 8209900. \\ E-mail: \{jmajin, hgomez, jflorez $\} @$ unicauca.edu.co.
}

\begin{abstract}
Resumen: Este artículo presenta el diseño, desarrollo e implementación de un guía para generar planes y programas de mantenimiento genérico a partir de la caracterización del proceso de mantenimiento basado en la filosofía TPM, la técnica RCM, en conceptos y enfoques de la norma ISO 9001:2008 y modelos de los estándares ANSI/ISA-95 y ANSI/ISA-88. De esta manera, se desarrolló una guía que permite, en primera parte, el diagnóstico, descripción, seguimiento, medición, y mejora del proceso de mantenimiento y del proceso productivo. Como segunda parte, la clasificación, identificación de equipos, plan y programación de mantenimiento. Para el caso de estudio, se evidenció una carencia en la primera puesto que el $88.24 \%$ de la población indica que el personal conoce las funciones operativas, y el $100 \%$ de ellas menciona la no existencia de orden cronológico al momento de realizar el mantenimiento y de un análisis de criticidad para sus equipos. Para la planta trilladora del caso de estudio se identificó los sistemas claves: sistema eléctrico, sistema mecánico y sistema de lubricación; Logrando la identificación y documentación de las diferentes etapas de proceso de apoyo que no estaban presentes en la gestión de procesos de la empresa. Como son inventarios, formatos, solicitudes de piezas e interacción y flujos de información de los distintos trabajadores y áreas de la empresa por los canales de comunicación adecuados. Conduciendo a la adecuación de los procesos misionales y estratégicos de la empresa.
\end{abstract}

Palabras clave: Mantenimiento, ISO 9001:2008, ANSI/ISA-95, ANSI/ISA-88, TPM, RCM, Gestión de procesos, Mejora continua.

Abstract: This paper have the design, development, and implementation of guide for plans and schedule of generic maintenance from the characterization of the maintenance process based on the Total Productive Management, RCM technique, in concepts and approaches to the ISO norm 9001:2008 and models of the ANSI/ISA-95 and ANSI/ISA88 standards. In this way, it was development a guide that permit, in the first part, diagnostic, description, tracking, measuring and improvement of maintenance process and the productive process. Like second part, the classification, equipment identification, plan and schedule of maintenance. For the study case, it shows a lack in the first part by that 
the $88.24 \%$ of the population indicates that the personal knows the operative functions, and $100 \%$ indicates the no existence of chronologic order in the moment to perform the maintenance and equipment criticality analysis. For thresher plant of the study case, it was indicate the key systems: electric system, mechanic system and lubrication system; achieving the identification and documentation of the different phases of support process that was not current in the process management of the company. Like that, inventories, formats, part requests, interaction, and information flow of different workers and company areas in adequate communication channels. Driving to the adequacy of the missional and strategic process of the company.

Keywords: Maintenance, ISO 9001:2008, ANSI/ISAA-95, ANSI/ISA-88, TPM, RCM, Process management, Continuous improvement.

\section{INTRODUCCIÓN}

En la actualidad se está afrontando retos constantes y competencia creciente en todo ámbito empresarial (AR Gomez, JCJ Fernández, 2018) (DC Rojas, OR Alvarado, 2017), por lo que cada empresa debe estar comprometida con la calidad (Verhoef, 2003); enfocar su misión y visión al crecimiento de la misma (Goetsch and Stanley, 2014). Para alcanzar esto se deben ejecutar distintos planes de acción dentro de la empresa. Todo esto se manifiesta con reducción de costos, mayor rentabilidad, mejoras en la productividad, motivación y compromiso por parte del personal en una cultura de calidad, y mejor posicionamiento en el mercado (Varley, 2014). Dichos planes de acción, se estructuran por medio de normas y procedimientos internacionales como lo son las normas ISO (Molina y Trujillo, 2008), en las cuales intervienen procesos de administración, de producción, financieros y de procesos de apoyo como el mantenimiento (Erkoyuncu et al, 2017), que ayudan a prevenir tiempos muertos en la producción (Botero y Álvarez, 2012), sustentados en la filosofía TPM (Total Productive Management) (Wakjira and Singh, 2012) junto con la metodología RCM (Reliability Centered Maintenance) (Anderson and Neri, 2012).

La aplicación de las normas ISO 9001, TPM y la RCM, tiene un gran alcance: siendo útiles para la gestión de procesos y de activos al interior de plantas industriales (Escobar, 2012); mejorando el mantenimiento preventivo industrial y la reducción de costos y paros en la producción (Villamil y Castillo, 2012); por ejemplo aplicando la metodología RCM en un plan existente de un sistema de embotellado, realizando una guía de la implementación para todo el personal de mantenimiento perteneciente a cada subsistema se notó una reducción del 15 al $20 \%$ en costos de mantenimiento correctivo (Poveda, 2012). Esto gracias a que la técnica RCM permite el análisis de causas de los estados de fallo y sus efectos, estableciendo una actividad de mantenimiento que elimine o reduzca los efectos de los fallos a un valor aceptable (Martínez, 2012). Otro claro ejemplo es la aplicación de un análisis de modo de fallo en una planta de concreto, por medio de una lista de chequeo y mejora de los instructivos de mantenimiento, sobre uno ya existente (Sanchez, 2012). Continuando con la temática de mantenimiento desde la perspectiva del estándar ISA- 95.00.03, se logra la conceptualización de mantenimiento con base al estándar, definiendo los modelos que contiene y enfatizándose en el modelo de actividades de la administración de operaciones de mantenimiento y los flujos de información (Viveros, 2013). Lo que también se ve reflejado en la evaluación del área de mantenimiento de una empresa textil revelando factores que afectan a esta área mediante el método propuesto por Dounce Villanueva para la solución de problemas de mantenimiento industrial (Guananga y Wilma, 2012). Y a un nivel contextualizado de la región, se logra un diagnóstico al interior de una empresa tostadora de café y propuesta del plan de mantenimiento preventivo, disminuyendo los paros en el proceso productivo, mejorando el área de mantenimiento, la gestión administrativa e información oportuna (Londoño, 2015). Ya en la aplicación de diagnóstico sobre el área de mantenimiento de una planta trilladora de café, se ejecuta un análisis del sistema de gestión, generando documentación técnica, gestión de repuestos y principalmente, su estado de equipos y mantenimiento (García, 2015). A pesar de la amplia aplicación de las metodologías y normas en las diferentes investigaciones, se evidencia en gran medida la carencia de análisis de criticidad o la profundidad de los mismos, junto con solo una reestructuración de planes de mantenimiento existentes, deficiencias en la formulación 
metodológica, y un corto rango de aplicación en las distintas industrias. Sin embargo, es una herramienta de gran utilidad para Colombia, ya que es un país fuerte en el sector cafetero en su aporte al PIB (Salcedo et al, 2013), pero algunas de sus industrias asociadas presentan tiempos improductivos de operación, planes de mantenimiento no adecuados o simplemente no los tienen.

Por ello con el fin de presentar la capacidad y rango de aplicabilidad de la norma ISO 9001:2008 (García, 2013), junto con estándares ANSI/ISA-88 (De Minicis et al, 2014) y ANSI/ISA-95.03 (Naedele et al, 2015); respecto al mantenimiento (Sivaram et al, 2008), en este artículo se presenta una guía genérica con estas normas aplicada a una planta trilladora de café pergamino de la empresa Caficauca (Popayán, Cauca-Colombia) (Majin y Gomez, 2015); con un análisis de criticidad, con aplicación de los pilares TPM y normas de mantenimiento adecuada (J Guzmán-Luna, ID Torres, 2017).

\section{METODOLOGÍA Y MATERIALES}

El método propuesto para el diseño y documentación del plan y programación de mantenimiento se organiza en una guía de mantenimiento que se soporta en los lineamentos de la norma de calidad ISO 9001:2008, estándares en automatización ANSI/ISA-88 y ANSI/ISA95, la técnica de modelado IDEF0 y el modelo clásico del ciclo PHVA o Deming. Estableciendo ocho etapas para desarrollar un plan y programación de mantenimiento genérico aplicable a cualquier empresa: diagnóstico; descripción, seguimiento, medición y mejora del proceso de mantenimiento; descripción del proceso productivo, clasificación e identificación de equipos, Plan de mantenimiento y Programación de mantenimiento.

\subsection{Diagnóstico del proceso de mantenimiento (DPM)}

Para conocer el estado actual del proceso de mantenimiento se realizan encuestas al personal involucrado. Ellas se estructuran a partir de preguntas dicotómicas, organizadas en seis ejes propuestos: TPM, RCM, planeación de mantenimiento, programación de mantenimiento, documentación del proceso y certificaciones de calidad.

\subsection{Caracterización del proceso de mantenimiento (CPM)}

Esta etapa abarca la descripción, seguimiento, medición y mejora del proceso de mantenimiento. Para ello se identifican rasgos distintivos estableciendo relación con otras actividades externas e internas del proceso como: entradas, salidas, proveedores, clientes y controles. Su objetivo es entender el funcionamiento y accionar del mismo para una adecuada toma de decisiones y mejora continua. Para eso, se determinan flujos de información, salidas, entradas, recursos y, finalmente, documentarlo en una ficha técnica, utilizando el método de modelado IDEF0 (Majin y Gomez, 2015) y algunas funciones que presenta el Modelo de Administración de Operaciones de Mantenimiento del estándar ISA 95.00.03, con lo que se plantea un modelo del proceso de mantenimiento genérico (PMPT). Luego, el PMPT es desagregado en cuatro sub-actividades: Planeación de Recursos de Mantenimiento, Organización y Asignación de Mantenimiento, Programación de Mantenimiento y Análisis de Mantenimiento. Se debe tomar en cuenta que en el PMPT hay dos tipos de flujo de información: data formal, información y requerimientos enviados desde el proceso de producción que pueden ser generados por las sub-actividades al interior del proceso, para realizar un trabajo de mantenimiento; y data informal, comunicación entre dos o más personas sobre un asunto en particular y que no queda plasmado en un documento, los cuales permiten un entendimiento de los flujos de información.

\subsection{Descripción del proceso productivo (DPP)}

El proceso de producción va ligando netamente al tema de mantenimiento al interior de las plantas industriales. Por tanto, se debe realizar una descripción del proceso de producción por etapas bajo un DFP (Diagrama de Flujo de Procesos). Éste permite establecer flujos de productos $\mathrm{y}$ subproductos y equipos principales, asignándoles un nombre descriptivo. Luego alineado al modelo físico de ANSI/ISA 88, se establece una jerarquía de equipos en términos de células, unidades y módulos de equipos, permitiendo contar con un inventario de activos de planta.

\subsection{Clasificación e identificación de equipos (CIE)}

Para una fácil localización de los módulos de equipos y su correcta referencia en órdenes de trabajo, bitácoras de mantenimiento y en formato de control de paradas de producción, se debe clasificar e identificar con códigos de ubicación a 
lo largo de la planta. A lo anterior, se suma el mejor manejo de cálculo de indicadores de mantenibilidad y disponibilidad de los equipos en planta.

\subsection{Plan de mantenimiento (PDM)}

El desarrollo del plan de mantenimiento se basó en dos componentes, mantenimiento preventivo y correctivo. Para este último se realizó una clasificación de equipos basado en un método de análisis de criticidad. Luego, bajo tres sistemas propuestos: lubricación, mecánicos y eléctricos se propuso un plan de mantenimiento preventivo (Wakjira and Singh, 2012).

\subsection{Programación de mantenimiento (PM)}

Determinadas las tareas de mantenimientos, se determina el orden en el que se efectúan los trabajos planificados tomando en cuenta: urgencia, materiales y disponibilidad de personal bajo un horizonte de tiempo de tres programas: maestro de mantenimiento: que cubre un período de un mes a 1 año, semanal: que cubre el trabajo a realizar en una semana, diario: que cubre el trabajo que debe realizarse cada día.

\section{ANÁLISIS Y RESULTADOS}

Con el fin de emplear la guía para la generación de planes de mantenimiento se procede a aplicar los 8 pasos planteados en una planta trilladora de café pergamino en el departamento del Cauca, caso de estudio, exponiendo los resultados más relevantes asociados a la guía de mantenimiento

\subsection{Diagnóstico del proceso de mantenimiento}

Con base en una serie de preguntas a la población $(\mathrm{P})$ conformada por Gerencia $(\mathrm{G})$, Jefes de áreas (JA) y Operarios(O) (ver Tabla 1); que interviene de manera directa e indirecta sobre el proceso de mantenimiento, se determinó el estado actual.

\section{Tabla 1: Encuesta del proceso de mantenimiento de la empresa}

\begin{tabular}{|c|c|c|c|}
\hline \multirow{2}{*}{ Eje } & Pregunta & \multicolumn{2}{|c|}{ Rta } \\
\cline { 3 - 4 } Filosofía TPM & $\begin{array}{c}\text { Si } \\
\text { ¿Los operarios están capacitados } \\
\text { para realizar las actividades de } \\
\text { limpieza, revisión y lubricación? } \\
\text { P }\end{array}$ & 18 & 0 \\
\cline { 2 - 4 } & $\begin{array}{c}\text { ¿Cuenta con inventario de } \\
\text { recursos? G }\end{array}$ & 1 & 17 \\
\hline
\end{tabular}

\begin{tabular}{|c|c|c|c|}
\hline & $\begin{array}{c}\text { ¿Se clasifica las herramientas de } \\
\text { acuerdo a su uso? O }\end{array}$ & 0 & 6 \\
\hline & $\begin{array}{l}\text { ¿Los equipos, herramientas y } \\
\text { repuestos poseen un código de } \\
\text { localización? O }\end{array}$ & 0 & 6 \\
\hline & $\begin{array}{c}\text { ¿Realizan un análisis de } \\
\text { criticidad? JA-O }\end{array}$ & 0 & 17 \\
\hline RCM & $\begin{array}{c}\text { ¿Sabe la función que cumple } \\
\text { cada equipo en el proceso } \\
\text { productivo? JA-O }\end{array}$ & 15 & 2 \\
\hline & $\begin{array}{c}\text { ¿Planean las actividades de } \\
\text { mantenimiento? P }\end{array}$ & 0 & 18 \\
\hline Planeación de & $\begin{array}{c}\text { ¿Cuenta con manuales de } \\
\text { equipos? JA-O }\end{array}$ & 0 & 17 \\
\hline Mantenimiento & $\begin{array}{c}\text { ¿Realizan mantenimiento } \\
\text { correctivo? JA-O }\end{array}$ & 17 & 0 \\
\hline & $\begin{array}{l}\text { ¿Realizan mantenimiento } \\
\text { preventivo? JA-O }\end{array}$ & 0 & 17 \\
\hline Programación & $\begin{array}{l}\text { ¿Las actividades de } \\
\text { mantenimiento obedecen a un } \\
\text { cronograma? JA-O }\end{array}$ & 0 & 17 \\
\hline Mantenimiento & $\begin{array}{c}\text { ¿La frecuencia de inspecciones } \\
\text { se realiza con base a algún } \\
\text { análisis de criticidad? JA-O }\end{array}$ & 0 & 17 \\
\hline & $\begin{array}{c}\text { ¿Existe fichas de los procesos de } \\
\text { mantenimiento? P }\end{array}$ & 0 & 18 \\
\hline & $\begin{array}{c}\text { ¿Existe Ficha técnicas de los } \\
\text { equipos? JA-O }\end{array}$ & 0 & 17 \\
\hline & $\begin{array}{c}\text { ¿Existe instructivo para la } \\
\text { realización de labores de } \\
\text { mantenimiento? JA-O }\end{array}$ & 0 & 17 \\
\hline & $\begin{array}{l}\text { ¿Existen formatos de control de } \\
\text { paradas de producción? JA-O }\end{array}$ & 0 & 17 \\
\hline $\begin{array}{l}\text { Documentación } \\
\text { del proceso de } \\
\text { mantenimiento }\end{array}$ & $\begin{array}{c}\text { ¿Existen formatos para } \\
\text { consignar averías de los } \\
\text { equipos? JA-O }\end{array}$ & 0 & 17 \\
\hline & $\begin{array}{c}\text { ¿Existen formatos para reportar } \\
\text { una avería o falla? JA-O }\end{array}$ & 0 & 17 \\
\hline & $\begin{array}{l}\text { ¿Existen órdenes de trabajo de } \\
\text { mantenimiento? JA-O }\end{array}$ & 0 & 17 \\
\hline & $\begin{array}{c}\text { ¿Existe indicadores de } \\
\text { mantenimiento? P }\end{array}$ & 0 & 18 \\
\hline & $\begin{array}{l}\text { ¿Existe formatos para realizar } \\
\text { reposición de repuesto de } \\
\text { materiales? P }\end{array}$ & 18 & 0 \\
\hline
\end{tabular}

Como resultado se destaca:

- Sobre el eje Filosofía TPM, el 100\% de la población sostiene que los operarios están capacitados para realizar las actividades de limpieza, revisión y lubricación; las herramientas de trabajo de la organización no se clasifican de acuerdo a su uso; los equipos, herramientas y repuestos no poseen código de localización. De otro lado, el $94.44 \%$ de la población afirma que la empresa no cuenta con inventario de recursos.

- En la Metodología RCM, el $100 \%$ de la población coincide en que no se ejecutan análisis de criticidad y el $88,24 \%$ de la población asegura que el personal de producción y mantenimiento conoce la función que cumple cada equipo en el proceso productivo. 
- Respecto a Planeación de Mantenimiento, el $100 \%$ de la población asevera que: Las actividades de mantenimiento no se planean; no existen manuales de equipo; no se realiza mantenimiento preventivo, pero si se emprenden acciones de mantenimiento correctivo.

- En Programación de Mantenimiento, el 100\% de la población afirma que las actividades de mantenimiento no obedecen a un cronograma, y la frecuencia de inspecciones no se realiza con base en análisis de criticidad.

- En relación a Documentación del proceso de Mantenimiento, el $100 \%$ de la población sostiene que no existen: fichas de los procesos de mantenimiento, ficha técnicas de los equipos, instructivos para la realización de labores de mantenimiento, formatos de control de paradas de producción, formatos para consignar averías de los equipos, formatos para reportar una avería o falla, formatos para realizar reposición de repuestos de materiales, órdenes de trabajo de mantenimiento ni indicadores de mantenimiento.

- Referente al eje Certificaciones de Calidad, el $100 \%$ de la población afirma que la organización se encuentra en el proceso de certificarse bajo norma NTC/ISO 9001-2008.

\subsection{Caracterización del proceso de mantenimiento}

Para la caracterización del proceso de mantenimiento en la empresa se parte del mapa o red de procesos general de la empresa. En este punto, la empresa del caso de estudio representó un desafío ya que no poseía, para algunos procesos, documentación y en otros, como producción y mantenimiento, eran nulos. Los procesos de toda empresa deben contribuir al desarrollo de la misión y visión, por lo que conviene identificarlos para que resulten significativos a través de un mapa de procesos. Al analizar y depurar las entrevistas con gerencia y otras áreas realizadas en el DPM permitieron establecer procesos: misionales, estratégicos y de apoyo.

Los procesos misionales para el caso de estudio, también, están ligados al cliente y al caficultor, permitiendo cumplir con la razón de ser de la organización, obtener un producto de calidad y economía social. En este sentido, los procesos incluidos como procesos misionales son: producción, facturación y ventas. Los procesos estratégicos son aquellos que están vinculados al ámbito de responsabilidades de la dirección, para el caso de estudio son: gerencia y planeación estratégica, los cuales están encargados de definir y establecer las metas, políticas y objetivos estratégicos. Los procesos de apoyo son aquellos que dan soporte a los procesos misionales, permiten a la empresa fortalecer los procesos misionales para lograr cumplir con los requisitos del cliente sobre el producto y proveer a ésta de los recursos necesarios para generar valor agregado que desean los clientes. Dentro de estos procesos se encuentran: Gestión administrativa y financiera, recursos humanos y salud ocupacional, gestión de compras, Mantenimiento, entre otros.

En el caso del Mantenimiento, proceso de interés, se procede a obtener el modelo de mantenimiento bajo la técnica de modelado IDEF0 (Fig. 1) identificando sus interfaces y flujos de información en relación con los otros procesos de la empresa (Majin y Gomez, 2015).

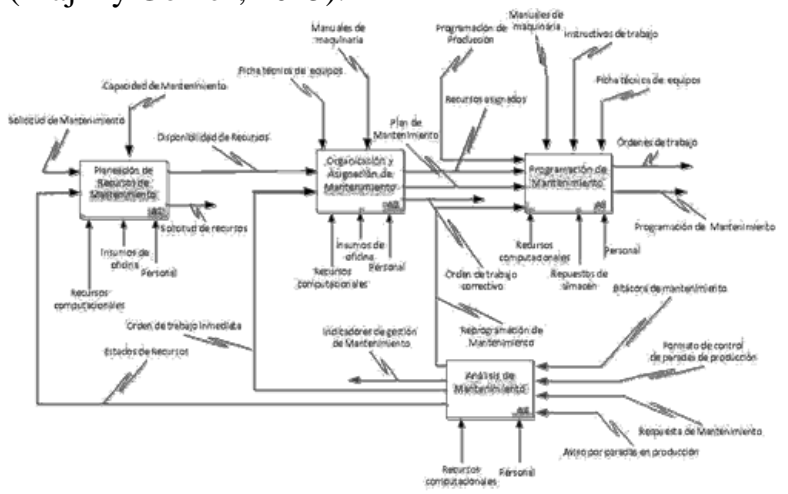

Fig. 1. Diagrama IDEFO(A-O) para el proceso de Mantenimiento propuesto para para la empresa.

\subsection{Clasificación e identificación de equipos}

Para el ordenamiento adecuado de los equipos es necesario en este caso de estudio efectuar un análisis de criticidad, realizando entrevistas estructuradas al personal de mantenimiento y operarios de producción para determinar la prioridad de los equipos más trascendentales en toda la planta de la empresa bajo la etiqueta: críticos, importantes y prescindibles (Majin y Gomez, 2015).

Equipos críticos: su parada o mal funcionamiento afecta directamente en los resultados de la empresa. En la empresa dichos equipos son Apolo IV que se encarga de la trilla y retrilla del café pergamino, y máquinas electrónicas encargadas de la selección del café verde.

Equipos importantes: aunque su parada o mal funcionamiento afecta la empresa, sus consecuencias son asumibles. En la empresa dichos equipos son Mesas densimétricas que detectan cambios ligeros en el grano de café y Catadoras, eliminan el grano que no tiene peso normal. 
Revista Colombiana de Tecnologías de Avanzada

Equipos prescindibles: su falla afecta escasamente los resultados, lo que implica bajos costos para su reparación. En la empresa dichos equipos son Bandas transportadoras de cárcamos y Elevadores, ambos se encargan de llevar la materia prima de un punto a otro.

\subsection{Plan y programación de mantenimiento}

Con base al diagnóstico y análisis de criticidad del CIE, se propone un plan de mantenimiento preventivo (PMPT) sobre los sistemas mecánicos, eléctricos y de lubricación, para cada uno de los equipos de la planta trilladora según el modelo físico ISA 88 (Majin y Gomez, 2015).

-Sistemas Mecánicos

El mantenimiento mecánico implica actividades como: inspecciones visuales, limpieza y revisión de equipos o elementos de los módulos de equipos con herramienta e instrumental adecuado. Se presenta el PMPT propuesto para la unidad de recepción y almacenamiento de café pergamino en silos (unidad A) (ver Tabla 2).

\section{Tabla 2: PMPT de los Equipos Mecánicos de la unidad A}

\begin{tabular}{|c|c|c|c|}
\hline Unidad & $\begin{array}{l}\text { Módulo de } \\
\text { equipo }\end{array}$ & $\begin{array}{ll}\text { Elementos } & \text { de } \\
\text { módulo } & \text { de } \\
\text { equipos } & \end{array}$ & $\begin{array}{c}\text { Labores } \\
\text { para los } \\
\text { elementos } \\
\text { de módulo } \\
\text { de equipos }\end{array}$ \\
\hline \multirow{10}{*}{ 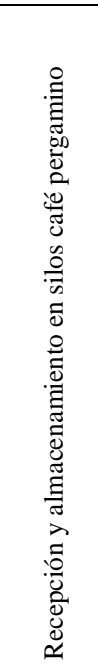 } & $\begin{array}{l}\text { Parrilla Principal } \\
\text { y Auxiliar }\end{array}$ & - & Limpiar \\
\hline & \multirow{7}{*}{$\begin{array}{l}\text { Elevador } \\
\text { cangilones } 1\end{array}$} & $\begin{array}{l}\text { Cangilones o } \\
\text { cubetas }\end{array}$ & $\begin{array}{l}\text { Inspeccionar } \\
\text { / Revisar }\end{array}$ \\
\hline & & $\begin{array}{ll}\text { Banda de } \\
\text { Cangilones }\end{array}$ & $\begin{array}{l}\text { Inspeccionar } \\
\text { / Revisar }\end{array}$ \\
\hline & & $\begin{array}{l}\text { Correa(marcha } \\
\text { y contra } \\
\text { marcha) }\end{array}$ & Revisar \\
\hline & & Rodamientos & $\begin{array}{l}\text { Inspeccionar } \\
\text { / revisar }\end{array}$ \\
\hline & & Poleas planas & Inspeccionar \\
\hline & & Motor & $\begin{array}{l}\text { Inspeccionar } \\
\text { /Revisar }\end{array}$ \\
\hline & & Chumaceras & $\begin{array}{l}\text { Inspeccionar } \\
\text { /Revisar }\end{array}$ \\
\hline & \multirow{4}{*}{$\begin{array}{ll}\text { Elevador } & \text { de } \\
\text { cangilones } 2 & \end{array}$} & $\begin{array}{l}\text { Cangilones } \mathrm{o} \\
\text { cubetas }\end{array}$ & Inspeccionar \\
\hline & & $\begin{array}{ll}\text { Banda } & \text { de } \\
\text { Cangilones } & \end{array}$ & Inspeccionar \\
\hline \multirow{2}{*}{ 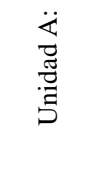 } & & $\begin{array}{l}\text { Correa(marcha } \\
\text { y contra } \\
\text { marcha) }\end{array}$ & Revisar \\
\hline & & Rodamientos & Inspeccionar \\
\hline
\end{tabular}

\begin{tabular}{|c|c|c|}
\hline & Poleas planas & Inspeccionar \\
\hline & Motor & Inspeccionar \\
\hline & Chumaceras & Revisar \\
\hline Silo 1 y silo 2 & - & $\begin{array}{l}\text { Limpiar } \\
\text { interna/exter } \\
\text { namente } \\
\end{array}$ \\
\hline \multirow{7}{*}{$\begin{array}{l}\text { Banda } \\
\text { transportadora de } \\
\text { la parrilla } \\
\text { principal }\end{array}$} & $\begin{array}{l}\text { Motor } \\
\text { reductores }\end{array}$ & $\begin{array}{l}\text { Inspeccionar } \\
\text { /Revisar }\end{array}$ \\
\hline & $\begin{array}{l}\text { Banda de tres } \\
\text { lonas }\end{array}$ & Inspeccionar \\
\hline & $\begin{array}{l}\text { Templetes de } \\
\text { banda }\end{array}$ & $\begin{array}{l}\text { Inspeccionar } \\
\text { /Revisar }\end{array}$ \\
\hline & $\begin{array}{l}\text { Desviadores de } \\
\text { banda }\end{array}$ & $\begin{array}{l}\text { Inspeccionar } \\
\text { /Revisar } \\
\end{array}$ \\
\hline & Chumaceras & $\begin{array}{l}\text { Inspeccionar } \\
\text { /Revisar }\end{array}$ \\
\hline & Poleas planas & $\begin{array}{l}\text { Inspeccionar } \\
\text { /Revisar }\end{array}$ \\
\hline & $\begin{array}{l}\text { Empates de } \\
\text { banda }\end{array}$ & Inspeccionar \\
\hline
\end{tabular}

\section{-Sistemas Eléctricos}

El mantenimiento eléctrico está encaminado a prever y asegurar el funcionamiento normal de los sistemas eléctricos, entre sus labores están: revisiones, limpieza y cambio de los componentes eléctricos. Se presenta el PMPT de los sistemas eléctricos de la unidad A (ver Tabla 3).

\section{Tabla 3: PMPT de los Equipos Eléctricos de la unidad $A$}

\begin{tabular}{|c|c|c|c|}
\hline Unidad & $\begin{array}{l}\text { Módulo de } \\
\text { equipo }\end{array}$ & $\begin{array}{l}\text { Elementos de } \\
\text { módulo de } \\
\text { equipo }\end{array}$ & $\begin{array}{l}\text { Labores para los } \\
\text { elementos de } \\
\text { módulo de } \\
\text { equipos }\end{array}$ \\
\hline \multirow{18}{*}{ 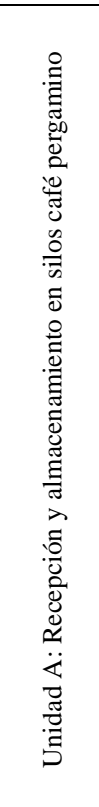 } & $\begin{array}{l}\text { Parrilla } \\
\text { Principal } \quad \text { y } \\
\text { Auxiliar }\end{array}$ & - & - \\
\hline & \multirow{9}{*}{$\begin{array}{l}\text { Elevador de } \\
\text { cangilones } 1\end{array}$} & \multirow{9}{*}{ Motor } & Limpiar \\
\hline & & & Revisar bornera \\
\hline & & & Cambiar bornera \\
\hline & & & Cambiar breaker \\
\hline & & & $\begin{array}{l}\text { Limpiar contactor } \\
\text { (es) }\end{array}$ \\
\hline & & & $\begin{array}{l}\text { Cambiar Contactor } \\
\text { (es) }\end{array}$ \\
\hline & & & Cambiar optimal \\
\hline & & & Cambiar térmico \\
\hline & & & Verificar amperaje \\
\hline & \multirow{8}{*}{$\begin{array}{l}\text { Elevador de } \\
\text { cangilones } 2\end{array}$} & \multirow{8}{*}{ Motor } & Limpiar \\
\hline & & & Revisar bornera \\
\hline & & & Cambiar bornera \\
\hline & & & Cambiar breaker \\
\hline & & & Limpiar contactor \\
\hline & & & Cambiar Contactor \\
\hline & & & Cambiar optimal \\
\hline & & & Cambiar térmico \\
\hline
\end{tabular}




\begin{tabular}{|c|c|c|}
\hline & & Verificar amperaje \\
\hline \multirow{3}{*}{ Silo 1 y silo 2} & \multirow[b]{2}{*}{ Lámpara } & Revisar \\
\hline & & $\begin{array}{ll}\text { Cambiar } & \text { tubo } \\
\text { fluorescente }\end{array}$ \\
\hline & Balasto & Cambiar \\
\hline \multirow{7}{*}{$\begin{array}{l}\text { Banda } \\
\text { transportador } \\
\text { a de la } \\
\text { parrilla } \\
\text { principal }\end{array}$} & \multirow{7}{*}{ Motor } & Limpiar \\
\hline & & $\begin{array}{l}\text { Revisar bornera y } \\
\text { Cambiar bornera }\end{array}$ \\
\hline & & Cambiar breaker \\
\hline & & $\begin{array}{l}\text { Limpiar y cambiar } \\
\text { contactor }\end{array}$ \\
\hline & & Cambiar optimal \\
\hline & & Cambiar térmico \\
\hline & & Verificar amperaje \\
\hline
\end{tabular}

- Sistemas de Lubricación

Los componentes mecánicos de los equipos están en continuo movimiento y se hace necesario recurrir a la lubricación para evitar desgaste de los elementos de los módulos de equipos o consumo excesivo de energía para el movimiento. Las actividades son: Lubricar con grasa o aceite y revisar grasa (consistente en inspecciones visuales sobre el estado de ésta). Se presenta el PMPT de lubricación de los sistemas mecánicos de la unidad A (ver Tabla 4).

Tabla 4: PMPT para la lubricación de los Equipos

\begin{tabular}{|c|c|c|c|}
\hline \multicolumn{4}{|c|}{ Mecánicos de la unidad A } \\
\hline Unidad & $\begin{array}{l}\text { Módulo de } \\
\text { equipo }\end{array}$ & $\begin{array}{ll}\text { Elementos } & \text { de } \\
\text { módulo } & \text { de } \\
\text { equipo } & \end{array}$ & $\begin{array}{c}\text { Labores para } \\
\text { los elementos } \\
\text { de módulo de } \\
\text { equipos }\end{array}$ \\
\hline \multirow{5}{*}{ 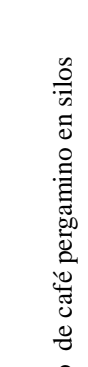 } & $\begin{array}{l}\text { Parrilla } \\
\text { Principal } \quad y \\
\text { Auxiliar }\end{array}$ & & \\
\hline & \multirow{2}{*}{$\begin{array}{l}\text { Elevador de } \\
\text { cangilones } 1\end{array}$} & Rodamientos & $\begin{array}{l}\text { Revisar } \\
\text { grasa/Lubricar }\end{array}$ \\
\hline & & poleas planas & $\begin{array}{l}\text { Revisar } \\
\text { grasa/Lubricar }\end{array}$ \\
\hline & \multirow{3}{*}{$\begin{array}{l}\text { Elevador de } \\
\text { cangilones } 2\end{array}$} & Rodamientos & $\begin{array}{l}\text { Revisar } \\
\text { grasa/Lubricar }\end{array}$ \\
\hline & & poleas planas & $\begin{array}{l}\text { Revisar } \\
\text { grasa/Lubricar }\end{array}$ \\
\hline \multirow{6}{*}{ 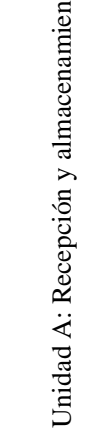 } & & Chumaceras & $\begin{array}{l}\text { Revisar } \\
\text { grasa/Lubricar }\end{array}$ \\
\hline & Silo 1 y silo 2 & & \\
\hline & \multirow{4}{*}{$\begin{array}{l}\text { Banda } \\
\text { transportadora } \\
\text { de la parrilla } \\
\text { principal }\end{array}$} & \multirow{2}{*}{$\begin{array}{l}\text { Motor- } \\
\text { reductores }\end{array}$} & $\begin{array}{l}\text { Completar } \\
\text { nivel de aceite }\end{array}$ \\
\hline & & & $\begin{array}{l}\text { Cambiar } \\
\text { aceite }\end{array}$ \\
\hline & & Chumaceras & $\begin{array}{l}\text { Revisar } \\
\text { grasa/Lubricar }\end{array}$ \\
\hline & & Rodamientos & $\begin{array}{l}\text { Revisar } \\
\text { grasa/Lubricar }\end{array}$ \\
\hline
\end{tabular}

Para la realización de programas de mantenimiento que no interfieran con la producción y con el aprovechamiento máximo de los recursos, se implementó una estrategia que consiste en preparar y programar el trabajo semanal. Esto se logra tomando en cuenta el análisis de criticidad donde se obtuvieron los tres sistemas base del caso de estudio y el plan de mantenimiento, donde se plantea el apoyo necesario a cada sistema. Además, se debe observar y registrar cada uno de los trabajos efectuados y realizando un análisis de cada uno de los equipos por medio de los métodos de mantenimiento.

Se propone diferentes convenciones de actividades para el PMPT para los equipos de la planta, el cual permite llevar un registro de éstas. Se indica en un formato de cronograma (ver Tabla 5) para las inspecciones realizadas mensual o diariamente por operarios de mantenimiento.

Tabla 5: Propuesta de actividades para la programación de mantenimiento preventivo

\begin{tabular}{|c|l|l|}
\hline \multicolumn{3}{|c|}{ COOPERATIVA DE CAFICULTORES DEL CAUCA } \\
\hline \multicolumn{2}{|c|}{$\begin{array}{l}\text { Programación de mantenimiento } \\
\text { preventivo anual }\end{array}$} & $\begin{array}{l}\text { Código } \\
\text { DOMA01 }\end{array}$ \\
\hline Columna & Actividad & Frecuencia \\
\hline \multirow{2}{*}{ C1 } & Actividad Diaria & AD \\
\cline { 2 - 3 } & Actividad Semanal & AS \\
\cline { 2 - 3 } & Actividad Mensual & AM \\
\cline { 2 - 3 } & Actividad Anual & AA \\
\cline { 2 - 3 } & Actividad Programada & AP \\
\hline \multirow{2}{*}{ C2 } & $\begin{array}{l}\text { La actividad implica } \\
\text { parada de producción }\end{array}$ & SI / NO \\
\hline \multirow{2}{*}{ C3 } & $\begin{array}{l}\text { La actividad implica } \\
\text { intervención externa }\end{array}$ & SI / NO \\
\hline \multirow{2}{*}{ C4 } & $\begin{array}{l}\text { La actividad implica } \\
\text { labor de taller } \\
\text { mecánico }\end{array}$ & SI / NO \\
\hline C5 & $\begin{array}{l}\text { La actividad se realizó } \\
\text { o se reprogramó }\end{array}$ & SI / NO \\
\hline
\end{tabular}

En la aplicación del modelo para el desarrollo de planes de mantenimiento, en el DPM el mapa de procesos que se obtuvo y propuso para el caso de estudio se abordó el proceso de mantenimiento y se desarrolló el diagrama IDEF0 (A-0) para éste, con lo que se identificó cuatro sub-actividades. Las cuales se establecieron bajo el modelo de actividades de administración de las operaciones de mantenimiento de ANSI/ISA-95.00.03, determinando 32 flujos de información (informal y formal) y se inventarió la herramienta necesaria para las labores de mantenimiento. En el CPM; al no existir información interna en el área de mantenimiento se planteó formatos como: Solicitud de Mantenimiento, solicitud de recursos, y formato de programación de mantenimiento, se desarrolló Indicadores de Gestión de Mantenimiento de disponibilidad y mantenibilidad y se propuso otros 
indicadores para realizar seguimiento y medición del proceso de mantenimiento. Ya en el DPP para la obtención de café excelso: Se elaboró la descripción de su proceso de producción, con su respectivo PFD y se levantó el Modelo Físico bajo ANSI/ISA-88 para los equipos de la planta del caso de estudio. Continuando en el CIE del Modelo Físico obtenido de la planta del caso de estudio, se inventarió y dividió el proceso en unidades, módulos de Propuesta de plan de Mantenimiento para la planta trilladora de la empresa. Llegando al PDM en el cual se elaboró una propuesta de plan de mantenimiento con base a las necesidades detectadas al interior del área de mantenimiento; desarrollándola bajo el Enfoque basado en Procesos de ISO 9001-2008, la cual presenta el qué hacer más no indica el cómo hacerlo; para suplir este interrogante, se utilizó los estándares en automatización industrial ANSI/ISA-95 y ANSI/ISA-88. Se asignó 130 labores de mantenimiento preventivo para 87 equipos de la planta según tres tipos: mecánico, eléctrico y de lubricación. Por ultimo en el PM con la realización del PDM preventivo se determinó la frecuencia de inspecciones, revisiones y otras actividades de mantenimiento preventivo. Esta frecuencia se determinó a partir del plan de producción obtenido de 2014, de la experiencia del jefe de mantenimiento y otros operarios de antigüedad. Se organizó de manera diaria, semanal, mensual y anual definiendo las actividades y tareas a realizar para cada uno de los elementos de módulos de equipo. Con lo que se pronostica que a partir del momento de aplicación de la propuesta de plan de mantenimiento se evitara paros innecesarios en los procesos productivos, lograr mayor disponibilidad de la planta y prolongar la vida útil de los equipos de producción. De manera paralela, se logró recopilar gran parte de la información del área de mantenimiento que no existía. Sin embargo, por la complejidad que representa el Modelo Físico de la planta del caso de estudio se realizó instructivo de trabajo para el compresor SULLAIR $40 \mathrm{H}$ de la unidad Selección Electrónica, para el motor del monitor de pergamino de la unidad pre limpieza y un instructivo general para la lubricación de los elementos de módulos de equipo de la planta. Además, se desarrolló Fichas técnicas para elevadores, catadoras, despedregadoras 1 y 2 , monitor de pergamino, monitor de almendra, mesas densimétricas, máquina trilladora apolo IV y bandas transportadoras de cárcamos.

\section{CONCLUSIONES}

Se diseñó e implementó una guía para generar planes de mantenimiento correctivo y preventivo acoplando las normas ISO 9001:2008, ANSI/ISA 88 , 95, la filosofía TPM y metodología RCM junto con un análisis de criticidad exhaustivo a una planta trilladora de la empresa Caficauca.

En este proceso fue de mayor impacto la formulación de la encuesta por medio de la filosofía TPM y su realización a la población, la cual ayudo a orientar de forma clara los siguientes pasos al momento de aplicar el plan de mantenimiento. De la población encuestada el 100 $\%$ notificó que no se realizaba análisis de criticidad, y al efectuar este en la planta trilladora se identificó 3 sistemas indispensables el sistema mecánico, el eléctrico y el de lubricación. Que a su vez permitieron establecer los procesos misionales de la empresa: producción, facturación y ventas; igualmente los estratégicos: gerencia y planeación estratégica, los cuales están encargados de definir y establecer las metas, políticas y objetivos estratégicos.

Se estructuró el proceso de mantenimiento como proceso de apoyo hacia los otros departamentos. Generando documentación de todas las subactividades como: formatos, solicitudes de repuestos, KPIs entre otros, adicional se identificaron las comunicaciones entre éstas presentando de forma clara y ordena el procedimiento frente a un mantenimiento preventivo o correctivo.

\section{RECONOCIMIENTO}

Por el apoyo presentado en la realización de esta investigación se agradece a la Universidad del Cauca, y a la empresa Caficauca.

\section{REFERENCIAS}

AR Gomez, JCJ Fernández, (2018), Revisión de la incorporación de la arquitectura orientada a servicios en las organizaciones. Revista Colombiana de Tecnologías de Avanzada ISSN: 1692-7257.

Verhoef, P. (2003). Understanding the effect of customer relationship management efforts on customer retention and customer share development. Journal of marketing 67.4, July, pp 30-45.

Goetsch, D., and Stanley B. D. (2014). Quality management for organizational excellence. Upper Saddle River, NJ: pearson.

Varley, R. (2014). Retail product management: buying and merchandising. Routledge. 
Molina, M.J. , y Trujillo E. F. (2015). Diseño de un sistema de gestión de calidad basado en la norma ISO 9001: 2008 en el Hospital de Especialidades San Juan HOSPIESAJ SA de la ciudad de Riobamba para el período 2013. BS thesis. Escuela Superior Politécnica de Chimborazo.

Erkoyuncu, J. A., et al. (2017). Improving efficiency of industrial maintenance with context aware adaptive authoring in augmented reality. CIRP AnnalsManufacturing Technology, January, pp 2033.

Botero, L. F. y Álvarez, M. E. (2012). Identificación de pérdidas en el proceso productivo de la construcción. Revista universidad EAFIT 39. Agosto, pp 64-78.

Wakjira, Workneh, and Singh, M. A. P: (2012). Total productive maintenance: A case study in manufacturing industry. Global Journal of Research In Engineering 12.1-G, May, pp 65-72.

Anderson, R. T., and Neri, L. (2012) Reliabilitycentered maintenance: management and engineering methods. Springer Science \& Business Media, 2012.

DC Rojas, OR Alvarado, (2017), Ontología para los sistemas holónicos de manufactura basados en la unidad de producción. Revista Colombiana de Tecnologías de Avanzada ISSN: 1692-7257.

Escobar, Luis. (2012). Estudio e implantación de un plan de mantenimiento y señalización en los equipos de la Planta Procesadora de Lácteos de la Asociación de Productores Alternativos San Pedro de Mulalillo del Cantón Salcedo en el año 2012. Universidad Técnica de Ambato, Ambato,Ecuador.

J. Guzmán-Luna, I. D. Torres, J. F. Alvarez. (2014). Propuesta de un generador de aplicaciones educativas basadas en televisión digital usando arquitectura de cómputo en la nube. REVISTA COLOMBIANA DE TECNOLOGÍAS DE AVANZADA, ISSN: 1692-7257. 1(23).

J. Guzmán Luna, I. Torres and J. Alvarez. (2014). Propuesta de un generador de aplicaciones educativas basadas en televisión digital usando arquitectura de cómputo en la nube. REVISTA COLOMBIANA DE TECNOLOGÍAS DE AVANZADA, vol. 1, no. 23, 2014.

Villamil, G. A. y Castillo, R. (2012). Diseño de un sistema de mantenimiento para el área de producción de Pasteurizadora Picos del Sicuara Ltda. Universidad la Sabana.

Poveda, A. J. (2012). Aplicación de la Metodología Mantenimiento del Sistema de llenado Automático de botellas de GLP. Escuela Superior Politécnica del Litoral.

Martínez, E. (2012). Mantenimiento Centrado en Confiabilidad Modos de Falla Análisis de Modos de Falla Y Efecto Fallas Funcional Contexto Operacional. Escuela Superior Politécnica del Litoral.

Sanchez, R. F. (2012). Elaboración de planes de mantenimiento preventivo para los equipos de las plantes de agregados. Univesidad Simon Bolivar, Sartenejas, Venezuela.

Viveros, P. et al. (2013). Propuesta de un modelo de gestión de mantenimiento y sus principales herramientas de apoyo. Ingeniare. Revista chilena de ingeniería 21.1. 125-138.

Londoño, J. A. (2015). Diseño del plan de mantenimiento programado Torre Granulado Colcafé Medellín. Instituto Tecnologico Metropolitano, Medellín, Colombia.

L.O. T. Tellez, M. P. S. Delgado, W. M. R.Contreras. (2014). Modelo de interventoría de tecnologías de información en el área de conocimiento de la gestión del alcance de pmbok ${ }^{\circledR}$ y alineado con iso 21500 y cobit@. REVISTA COLOMBIANA DE TECNOLOGÍAS DE AVANZADA, ISSN: 1692-7257. 1(23).

Tangarife Tellez, L., Sánchez Delgado, M., \& Rojas Contreras, W. (2014). Modelo de interventoría de tecnologías de información en el área de conocimiento de la gestión del alcance de pmbok ${ }^{\circledR}$ y alineado con iso 21500 y cobit@. REVISTA COLOMBIANA DE TECNOLOGÍAS DE AVANZADA, 1(23).

García, A. B. (2015). Diseño de un plan de mantenimiento preventivo para la empresa Carcafé Ltda. Universidad Tecnológica de Pereira, Pereira, Colombia.

Salcedo, L. Pinzón, R. y Duarte C. (2013). El paro nacional agrario: un análisis de los actores agrarios y los procesos organizativos del campesinado colombiano. Centro de Estudios Interculturales, julio, pp 12-20.

García, V. M. et al. (2013). Implantación de un sistema de gestión de calidad según norma UNE-UN-ISO 9001: 2008 en un servicio de medicina nuclear Revista Española de Medicina Nuclear e Imagen Molecular 32.1, septiembre, pp 1-7.

De Minicis, M. et al. (2014). Recipe Development Process Re-Design with ANSI/ISA-88 Batch Control Standard in the Pharmaceutical Industry. International 
Journal of Engineering Business

Management, december, pp 6-16-21.

Naedele, M. et al. (2015). Manufacturing execution systems: A vision for managing software development. Journal of Systems and Software 101. 59-68.

Sivaram, N. M. et al. (2014). Synergising total productive maintenance elements with ISO 9001: 2008 standard based quality management system. The TQM Journal 26.6. 534-549.

Majin J. W. y Gomez, H. Propuesta de plan de mantenimiento para la planta trilladora de la Cooperativa de Caficultores del Cauca. Universidad del Cauca, 2015. 